\title{
Self-configuring Software-defined Overlay Bypass for Seamless Inter- and Intra-cloud Virtual Networking
}

\author{
Kyuho Jeong \\ ACIS Lab - ECE Department \\ University of Florida \\ Gainesville, Florida, USA \\ kyuhojeong@acis.ufl.edu
}

\author{
Renato Figueiredo \\ ACIS Lab - ECE Department \\ University of Florida \\ Gainesville, Florida, USA \\ renato@acis.ufl.edu
}

\begin{abstract}
Many techniques have been proposed to provide, transparently, the abstraction of a layer-2 virtual network environment within a provider, e.g. by leveraging Software-Defined Networking (SDN). However, cloud providers often constrain layer-2 communication across instances; furthermore, SDN integration and layer-2 messaging between distinct domains distributed across the Internet is not possible, hindering the ability for tenants to deploy their virtual networks across providers. In contrast, overlay networks provide a flexible foundation for inter-cloud virtual private networking (VPN), by tunneling virtual network traffic through private, authenticated end-to-end overlay links. However, overlays inherently incur network virtualization overheads, including header encapsulation and user/kernel boundary crossing. This paper proposes a novel system - VIAS (VIrtualization Acceleration over SDN) - that delivers the flexibility of overlays for inter-cloud virtual private networking, while transparently applying SDN techniques (available in existing OpenFlow hardware or software switches) to selectively bypass overlay tunneling and achieve near-native performance for TCP/UDP flows within a provider. Architecturally, VIAS is unique in how it integrates SDN and overlay controllers in a distributed fashion to coordinate the management of virtual network links and flows. The approach is self-organizing, whereby overlay nodes can detect that peer endpoints are in the same network and program bypass flows between OpenFlow switches. While generally applicable, VIAS in particular applies to nested VMs/containers across cloud providers, supporting seamless communication within and across providers. VIAS has been implemented as an extension to an existing virtual network overlay platform (IP-over$\mathrm{P} 2 \mathrm{P}$, IPOP) by integrating OpenFlow controller functionality with distributed overlay controllers. We evaluate the performance of VIAS in realistic cloud environments using an implementation based on IPOP, the RYU SDN framework, Open vSwitch, and LXC containers across various cloud environment including Amazon, Google compute engine, and CloudLab.
\end{abstract}

Permission to make digital or hard copies of all or part of this work for personal or classroom use is granted without fee provided that copies are not made or distributed for profit or commercial advantage and that copies bear this notice and the full citation on the first page. Copyrights for components of this work owned by others than ACM must be honored. Abstracting with credit is permitted. To copy otherwise, or republish, to post on servers or to redistribute to lists, requires prior specific permission and/or a fee. Request permissions from permissions@ acm.org.

HPDC'16, May 31-June 04, 2016, Kyoto, Japan

(C) 2016 ACM. ISBN 978-1-4503-4314-5/16/05 . \$ $\$ 15.00$

DOI: http://dx.doi.org/10.1145/2907294.2907318

\section{Keywords}

SDN; OpenFlow; Cloud Computing; virtualization; P2P; overlay network

\section{INTRODUCTION}

Since the rise of multi-tenant clouds, the approach of providing virtualized computing resources such as virtual machines and containers has become key to an increasing body of distributed computing applications. Today, computing resources are readily available through multiple cloud service APIs and portals, and can be customized and instantiated from a template image dynamically. Cloud providers expose such virtual resources with a set of instance types, with discrete combinations of CPU, memory and storage capacities. While cloud platforms also offer virtual network capabilities (e.g. Amazon Virtual Private Cloud, OpenStack Neutron), these do not extend across multiple providers. Tenants who wish to benefit from the ability to choose the best offerings from multiple cloud providers, dynamically, must deal with different APIs and virtualization platforms (e.g. KVM, Xen), and providers who do not inter-operate in their virtual network capabilities. This paper proposes VIAS, a virtual network that addresses the challenge of providing a consistent virtual network abstraction across cloud instances deployed on multiple providers.

The increasing variety of cloud providers and the added flexibility of an extra layer of indirection from nested virtualization $[21,35,2]$ are welcome news for tenants interested in deploying multi-cloud virtual infrastructures that seek to achieve high availability, geographical distribution, and to minimize cost/performance (while avoiding vendor lock-in) by selecting the best instance for a task from a variety of offerings of reserved and spot instances in the cloud market. Networking, however, becomes harder - it requires the coordinated configuration of virtual network interfaces, switches and routers across multiple instances. Furthermore, each cloud infrastructure has its own networking model and stack, and may be subject to connectivity constraints such as network address translation (NAT), in particular in private clouds. While tenants can use cloud APIs and Web interfaces within a provider to configure the network for their cloud instances (e.g. boto [1] or gcloud [9]), there is no inter-operability across providers. This leads to management complexity that is greatly compounded when tenants distribute their applications across clouds and expect elastic provisioning and management operations, such as VM migration, to work seamlessly.

Several applications can benefit from inter-cloud deploy- 
ments. For instance, workflow management system such as Pegasus [5] can use heterogeneous resources to perform different tasks, and the best cost/performance resources may not be co-located in the same provider. In high-throughput applications such as parameter sweeps and bag-of-tasks, the most cost-effective computing resources, which are harvested upon the completion of a single task and reassigned to the other tasks [18], also may not be co-located.

Nonetheless, while applications can benefit from the flexibility cost/performance benefits of choosing resources across multiple providers, inter-cloud deployment can significantly increase the complexity of configuring, deploying and managing applications. Network virtualization provides a basis to address this challenge. Techniques for network virtualization based on overlay tunneling and encapsulation at endpoints, and Software-Defined Networking (SDN) at the network fabric have both been explored in related work, in different contexts.

Overlay networks have been applied to network virtualization (at layers 2 or 3 ) across cloud providers. For example, hSwitch [7] forms an overlay network by creating Generic Routing Encapsulation (GRE) tunnels across dispersed nested VMs. However, because it creates tunnels based on provided configuration files, the overlay topology is static, and dynamic joining/leaving of instances is complex to manage. The IP-over-P2P (IPOP [12]) overlay supports both layer 2 and 3 virtual private networking with peer-to-peer tunnels that are dynamically created and managed - even when nodes are behind NATs. Tunnels are created to reflect relationships stored in messaging or online social network services (e.g. an XMPP server), supporting overlay topologies such as unstructured social graphs and structured P2P networks [28]. IPOP has been used in the Kangaroo [21] inter-cloud platform-as-a-service system; however, its overlay processing hindered the performance of nested VMs across hosts within a provider. This paper directly addresses this performance limitation.

Another approach to network virtualization comes from the service providers. Techniques such as VLANs are not able to cope with the need for flexible configuration and isolation across large numbers of tenant address spaces, nor do they have sufficient range of virtual private address subnets [13]. The advent of SDN techniques and the OpenFlow standard have unlocked the potential to address these limitations and deploy network virtualization services at scale within service providers. However, while SDN-based solutions are becoming well-understood within the context of a single provider's infrastructure, SDN solutions across noncooperating providers (e.g. different public/private clouds) are currently not feasible, as providers are not willing to allow external entities to exert control in their SDN infrastructure.

Overlays are scalable and general-purpose, but suffer from performance degradation within a data center, while SDNbased techniques are difficult to deploy across providers, because cloud resources (i.e. VMs) are managed by independent entities (possibly constrained by the presence of NATs), and establishing authenticated, encrypted links in the presence of dynamic instantiation and resource migration is complex, and requires coordination.

This paper presents VIAS, a novel approach that integrates overlay and SDN techniques to support flexible and high-performance virtual networking, in particular across

\begin{tabular}{|c|c|c|c|c|c|c|c|c|c|c|c|}
\hline \multicolumn{12}{|c|}{ Multitenant Applications } \\
\hline nVM & nVM & nVM & $\mathrm{nVM}$ & $\mathrm{nVM}$ & nVM & nVM & nVM & nVM & $\mathrm{nVM}$ & nVM & nVM \\
\hline \multicolumn{12}{|c|}{ Layer 2 Virtualization Network for Nested VM } \\
\hline \multicolumn{2}{|c|}{ VM } & \multicolumn{2}{|c|}{ VM } & & & \multicolumn{2}{|c|}{ VM } & & M & \multicolumn{2}{|c|}{ VM } \\
\hline \multicolumn{4}{|c|}{ Amazon EC2 } & \multicolumn{4}{|c|}{ Google Compute Engine } & \multicolumn{4}{|c|}{ CloudLab } \\
\hline
\end{tabular}

Figure 1: Illustration of layer-2 network virtualization for nested VMs across three cloud providers. The goal is to allow distributed applications (possibly multi-tenant) within nVMs to seamlessly communicate, as if they were connected to the same layer- 2 segment - even though they are distributed across independently-managed providers.

tenant-managed nested virtualization instances distributed across cloud providers. It does so while exposing a network layer 2 abstraction to endpoints. VIAS supports the general case (the abstraction of a layer-2 virtual private networking linking instances across providers) by employing overlay networking as the substrate for virtualization, and optimizes for a common case (network flows among nodes within the same provider) by means of a novel performance enhancement technique of automatically detecting and programming fast bypath TCP/UDP flows using SDN APIs. Such SDNprogrammed VIAS bypass removes the necessity of packet encapsulation and delivers virtualization performance near wire link speed. VIAS detects traffic-intensive TCP/UDP flows inside the overlay-encapsulated data traffic, and automatically switches over to the SDN fabric whenever such path can be programmed - such as when endpoints are within the same cloud provider.

The main contribution of this paper is a novel system that integrates distributed overlay network and SDN controllers to self-configure peer-to-peer virtual private overlay network tunnels across cloud providers, and transparently detect and program virtual network bypath flows within a provider. The system has been implemented by leveraging an existing overlay technique (IPOP) as a substrate, and integrating existing SDN programmable switches (Open vSwitch) to establish bypass flows. While it can generalize to different overlay tunneling and SDN targets, VIAS applies, in particular, to inter-cloud nested virtualization environments, where tenants can deploy software SDN switches (e.g. Open vSwitch) in their own instances. The paper demonstrates the functionality of VIAS within and across clouds, and evaluates the performance of our prototype in multiple cloud environments.

The rest of this paper is organized as follows: Section 2 elaborates on the use of overlay network and SDN as network virtualization solutions. Section 3 describes the general architecture of VIAS. Section 4 provides details on the design and implementation of a VIAS system. Section 5 presents results from the evaluation of our prototype in realistic cloud environments. Section 6 discusses related work, and Section 7 concludes the paper.

\section{BACKGROUND}

This section overviews techniques, issues and challenges that serve as a basis to motivate the design and uses of VIAS. 


\subsection{Network Virtualization}

The purpose of VIAS is to provide a complete layer $2 \mathrm{ab}-$ straction to resources deployed across heterogeneous cloud services (as illustrated in Figure 1), without significant performance degradations. In general, there are two approaches to tackle this issue. The first relies on tenants deploying their own overlay network - which has the key advantage of not requiring any support from the underlying infrastructure. The other approach is to exploit SDN and/or network function virtualization (NFV) services provided by the cloud provider - which has the disadvantage of lack of interoperability across providers.

Overlay network: In overlay networks and VPNs (Virtual Private Network), the entire header and payload of a virtual network packet are encapsulated (and possibly encrypted and authenticated) by another layer to transfer the packet over public network links. Tunneling techniques such as L2TP, GRE or MPLS take advantage of encapsulation, which prepends an additional network header to the same or different OSI layer of the packet [27]. Tunnels can be built as stand-alone point-to-point links, or organized to form a topology, such as a mesh or structured P2P, that can be used for scalable overlay routing $[34,11,12]$. Overlay and tunneling techniques benefit from the flexibility of using encapsulation at the endpoints - which does not require changes to the infrastructure - but suffer from performance degradation. The additional encapsulation header is a source of overhead, limiting the effective maximum transmission unit (MTU) size. Furthermore, overlay processing adds computation overhead of dealing with encapsulation - possibly at the user level, as typical overlay networks are implemented as user-level processes.

SDN: Software Defined Networks initially emerged from the necessity of testing experimental protocols to overcome the difficulties of deploying new protocols on legacy hardware switches and routers [16]. Subsequently, the need for efficiently supporting large multi-tenant enterprise data centers, such as public and private clouds, has motivated the adoption of SDN techniques as an approach complementary to NFV (Network Function Virtualization) for data center network virtualization. Virtualization in cloud computing impacts the network performance because of its inherent sharing of processor resources. This can lead to negative impact on network performance and stability, such as exceptionally long delays and degraded throughput [31]. SDN and NFV techniques can mitigate performance degradations by migrating network virtualization processing overheads to network devices, and possibly lead to substantial reductions in operating expense for cloud and network service providers [10]. For example, VMware [13] supports network virtualization through both logically and physically deployed SDN nodes, providing a network virtualization platform (NVP) within a multi-tenant data center. NVP leverages software switches on VMware hypervisors at each server endpoint.

Typically, tunneling and SDN approaches are used in different network virtualization contexts - typically across (tunneling) and within (SDN) data centers. VIAS seeks to integrate these two approaches, by building a tunneled overlay network layer as a basis for layer-2 virtualization within and across providers, and by mitigating performance degradation by selectively applying SDN to establish a bypass path for intra-cloud TCP/UDP flows.

\subsection{Nested Virtualization}

While VIAS is generally applicable to any cloud infrastructure that exposes a virtual network interface (e.g. a tap device) and SDN switch (e.g. Open vSwitch), it complements, in particular, nested virtualization techniques. A nested virtualized environment inter-connected by VIAS enables users to deploy flexible inter-cloud virtual platforms that allow for a consistent application environment across different providers, and flexible fine-grain management of nested VM resources.

In its most general form, nested virtualization allows the capability of virtual machines to themselves host other virtual machines, in a recursive fashion. It builds upon the ability of "classic" VM monitors/hypervisors to expose the abstraction of a virtual machine at the instruction set architecture (ISA) layer, and can be supported if the underlying ISA follows the guidelines set forth in seminal virtualization work [20]. Initially applied in partitionable mainframe systems, nested virtualization became feasible in commodity server systems with the advent of virtualization extensions for the x 86 architecture [30], and has been motivated by use cases in cloud computing [2].

While the most general form of nested virtualization allows a "classic" hypervisor to be instantiated within a virtual machine and supports completely unmodified software to run within VMs (e.g. instantiating KVM within VMware), the nested approach can also be applicable in other configurations, such as nested para-virtualized hypervisors (e.g. Xen on Xen [32]), trading off potential performance benefits with the additional requirement of software modifications in the kernel and/or applications. In particular, a form of nested virtualization that is appealing for deployment of software services in IaaS cloud computing platforms is to use the hypervisor of the cloud provider (e.g. Xen in Amazon EC2, KVM in Google compute engine, Hyper-V in Microsoft Azure) to deploy O/S containers (e.g. Linux LXC/Docker). This is the approach taken in Kangaroo [21]; the key advantage of this approach is performance, because containers are light-weight. The requirement to run software within containers poses a constraint, as nested instances need to use the same O/S kernel, but it is acceptable in many applications, as the adoption of container technologies continues to increase.

Different approaches to nested virtualization, as described above, yield different models of how virtual CPUs, memory and storage are allocated and managed. For instance, classic VMs expose virtual CPUs, physical memory, and block storage devices, while containers expose processes, virtual memory, and file systems at the O/S layer. Nonetheless, in general, nested virtualized systems typically expose a similar interface to the networking subsystem across multiple platforms. The lower-layer has Ethernet virtual network interface(s) provided and managed by a cloud provider's "outer" hypervisor; these are multiplexed among Ethernet virtual network interfaces managed/exposed by the tenant's "inner" virtualization stack.

Throughout this paper we use the term "nested VMs" (or "nVMs" in short) to refer broadly to any nested virtualization technique that exposes a layer-2 virtual network interface to each instance. In our evaluation, we focus on $\mathrm{O} / \mathrm{S}$ containers as nVMs; nonetheless, the layer 2 virtual networking techniques in VIAS generally apply to any virtualized system that uses layer-2 Ethernet virtual interfaces. 


\subsection{Networking in Nested Containers}

Nested virtualization supports virtual network interfaces for nested instances. For instance, Linux containers (LXC) expose one or more TUN/TAP devices for each container. By default, LXC also creates a Linux bridge on the host of a provisioned VM, and connects all virtual network interfaces of the nested VMs to this bridge. In this way, all the nested VMs attached to this bridge reside on the same layer-2 Ethernet segment. Because multiple nested instances share a single interface of the lower-layer hypervisor, it is necessary to assign and manage addresses, and multiplex access to the single interface using address translation. To this end, LXC also creates Network Address Translation (NAT) rules in the host machine, using iptables. To support automatic address assignment, LXC runs a lightweight DHCP server on the host machine. Thus, upon instantiation, each nested VM gets assigned a random IP address within a predetermined subnet range of (private) IP addresses. While not all nested virtualization technologies automate the process of providing a layer 2 network environment behind a virtual NAT, this behavior can be programmed using existing Linux devices and toolsets - a virtual bridge, iptables and dnsmasq.

\subsection{NAT Traversal}

In general, when deploying resources across clouds, tenants may be required to deal with private addresses and network address translation (NAT). First, public IPv4 addresses are scarce, and typically come with a price premium. For instance, EC2 provides by default a private IP address and a NAT-mapped public IP address, and charges extra for persistent public IP addresses (elastic IPs). Second, with deeper, nested virtualization, network interfaces provisioned by a cloud provided are further mutiplexed, as nested VMs are given private addresses. It is also possible that the VMs provisioned by a service themselves have private IP addresses that are translated by a NAT device, which is common in private clouds.

Consider, for instance, a tenant using a VM provisioned by a cloud provider (cVM1), and then instantiating nested instances (nVM1...nVMi) within cVM1. Each nVM is instantiated by the tenant, and hence has a virtual address that is private. While the $\mathrm{nVMi}$ instances can communicate within cVM1 through a virtual bridge or switch, in order to communicate across multiple instances (e.g. to $\mathrm{nVMs}$ hosted in other machines in the same provider, or on a different provider), it becomes necessary to map and translate addresses. In the absence of the ability to provision public addresses to nested VM instances (which is currently not offered by cloud providers), network virtualization techniques must deal with multiplexing and translation of nested/host addresses. This can, in principle, be accomplished through careful crafting of network rules by the tenant. However, this becomes complex and error-prone as the network increases in size and nodes are elastically added/removed from a tenant's infrastructure.

VIAS leverages the ICE [24], STUN [25] and TURN [15] protocols integrated in IPOP [12] to support dynamic NAT traversal, allowing (nested) cloud instances across different providers and behind one or more levels of NAT to establish P2P overlay links for tunneling. The key principle taken in VIAS is that NAT traversal is not considered as an exception, but rather a lowest-common denominator in estab-

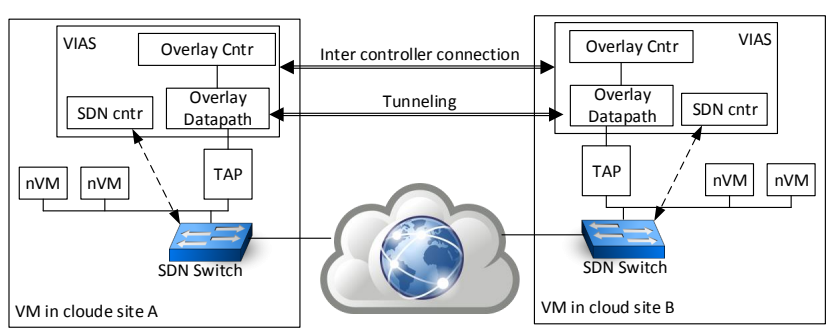

Figure 2: VIAS overview. VIAS main modules are userlevel applications that are responsible for managing bypass flows (SDN cntr), setup and configuration of overlay tunnels (Overlay cntr), and encryption, encapsulation, tunneling and NAT traversal (Overlay datapath). The VIAS overlay datapath binds to an SDN switch port through a virtual network interface (tap), and the SDN controller module programs the switch. The SDN switches are commodity software/hardware devices programmed using OpenFlow APIs; they are also referred to as vias-nat-switch in the paper.

lishing inter-cloud overlay links. VIAS thus bootstraps from $\mathrm{P} 2 \mathrm{P}$ links that can handle multiple levels of NATs across providers, and tunnel layer-2 virtual network traffic as the general case; for the particular case where P2P links are established within a single provider, the overhead of NAT traversal is side-stepped by identifying and establishing SDN bypass flows for TCP/UDP communication.

\section{VIAS OVERALL ARCHITECTURE}

The key design requirements in VIAS are: 1) to expose a layer-2 abstraction to unmodified applications, 2) to operate as a user-level application that can be deployed in any existing cloud instance, thus not requiring development of kernel modules, 3) to support private, encrypted tunnels in inter-cloud communications, and 4) to avoid the overhead of encapsulation and kernel/user crossings for TCP/UDP flows within a cloud provider. The VIAS architecture addresses these requirements by: fully supporting layer-2 tunneling through TLS/DTLS P2P overlay links as a baseline functionality implemented in user-level overlay software, and by automatically detecting and programming SDN switches to bypass TCP/UDP flows.

There are important reasons why VIAS bypasses TCP/UDP flows via SDN switches, but carries other virtual network traffic (e.g. ARP, ICMP) in tunnels. First, TCP/UDP are the transports used by the majority of cloud applications and the common case that needs to made fast. Second, cloud providers typically allow instances to communicate using TCP/UDP, but block layer-2 protocols (such as ARP); since we only assume SDN switches to be available at the endpoint at the minimum (e.g. software switches such as Open vSwitch in an instance), VIAS is not necessarily able to program the cloud provider's entire switch fabric.

With existing OpenFlow standard APIs and existing functionality in cloud platforms, bypass flows can be programmed by coordinated mapping/translation of TCP/UDP flow endpoints at SDN switches conencted to VIAS virtual network interfaces. To accomplish this, the VIAS architecture is structured as illustrated in Figure 2. It comprises of three main modules. 
SDN Controller: This module acts as a controller for the SDN switches. In essence, it allows OpenFlow switches to retain conventional features (such as MAC learning) but, in addition, implements the distinctive features of VIAS: the abstraction of a layer-2 virtual network, and isolation of the virtual network from the host network. The VIAS SDN controller programs gateway and NAT functionalities on the SDN switch, such that virtual network endpoints can have a different subnet and private address range from the host network's. Those features are programmed using standard OpenFlow APIs, without any modifications to the switch, allowing the use of commodity hardware and software implementations of SDN switches. The main requirement is that the VIAS module is allowed to issue OpenFlow API calls to the switches. For brevity, we will refer to the SDN switches with these capabilities as vias-nat-switch.

The vias-nat-switch also implements VIAS overlay bypass to achieve high throughput for intra-cloud TCP/UDP flows. These flow bypass rules are programmed (also using OpenFlow APIs) to have higher priority than that of other flow rules. We give more details on the implementation (and limitations) of this vias-nat-switch and overlay bypass in Subsections 4.1 and 4.3 .

Overlay datapath: Packets captured by the tap virtual network interface are handled by this module. This module runs as a user-space process that reads/writes from the tap device, and executes all low-level packet processing functions, such as prepending or translating network headers, as well as encryption and authentication. VIAS leverages the IPOP [12] overlay stack for this module. It reads the destination address to look up an overlay destination, prepends IPOP and UDP headers, then injects the packet to the WAN interface.

While the creation and termination of $\mathrm{P} 2 \mathrm{P}$ overlay links are managed by the overlay controller, the metadata associated with each P2P overlay link (such as peer UID, IPv4 or MAC addresses) are used by the overlay datapath module to make forwarding decisions. Likewise, the necessary attributes of these headers (such as tunneling identifier and mapped IP and port numbers) are dynamically assigned by the overlay controller and programmed into the overlay datapath module. After the UDP header prepending, packets are ready to traverse through tunnels across the public Internet. We elaborate on the design and implementation of this module in Subsection 4.2.

Overlay Controller: The creation and termination of P2P links among overlay nodes, and the topology of the overlay network, are managed by this module. The VIAS overlay controller extends the IPOP overlay controller, which currently supports three types of topologies. One is an unstructured social network graph topology, where P2P links are created based on social links. A second topology is an allto-all graph connecting all nodes, while the third topology is based on the Chord [28] structured P2P system. VIAS can use the all-to-all topology for small networks (tens of hosts), or the structured topology to scale to larger nodes. In the structured topology, each node identifier is based on SHA-1 of its virtual IP address, and identifier-based structured routing is performed when there is no direct $\mathrm{P} 2 \mathrm{P}$ link connecting nodes. Structured topology policies, such as the number of successors or chord links, are configurable in the controller.

Overlay link/bypass flow management: The gen- eral approach taken by VIAS for the management (creation, monitoring, and tear-down) of links is as follows. First, overlay links are created by the overlay controller. Links may be created to enforce a topology invariant (e.g. left/right neighbors and chords in structured P2P), or on-demand based on traffic initiated by a peer. Second, for active links, their state is monitored (with respect to their traffic, and the transport addresses) by the overlay datapath module, and made available to the overlay controller. Building on overlay link monitoring mechanisms, the overlay controller defines policies for establishing a bypass TCP/UDP flow that takes into account: 1) the traffic between nodes, and 2) whether the two endpoints are within the same provider network, to initiate a link a request to create a bypass flow. The mechanisms to initiate a bypass flow are handled by the SDN controller module.

There are two instances where a bypass flow might be terminated: one is after VM migration, and another is if resources (available ports) are exhausted at the SDN switch. The policies to deal with bypass termination are left for future work, but mechanisms available to support these policies are monitoring of traffic per flow (which can be used to prioritize high-throughput traffic), and migration events (e.g. unsolicited ARP requests) to notify controllers to terminate a flow. In such events, the SDN controller can then clear out bypass rules, hence reverting all virtual network traffic to pass through overlay tunnels.

\section{IMPLEMENTATION}

In this section, we provide details of a VIAS prototype implementation, highlighting how each module is implemented and integrated. Moreoever, we explain how the transition between overlay and SDN virtualization takes place in VIAS. In Subsection 4.1, we elaborate on how the dynamic NAT feature offered by VIAS is programmed into the OpenFlow SDN switch. In Subsection 4.2, we explain tunneling and overlay virtualization along with various tunneling modes. Finally, in Subsection 4.3, we describe how VIAS detects flows and implements rules to bypass overlay virtualization by using SDN flow rules.

\subsection{SDN Controller}

As explained in Section 2.3, each nested VMs should be presented the full abstraction of a private layer 2 network, while still able to access the public Internet as if behind a NAT. To support this requirement, VIAS programs address translation rules using the OpenFlow controller, which makes Open vSwitch (or any other OpenFlow enabled devices) to work as a full-cone NAT router.

Through a configuration file, VIAS specifies a single switch port as its WAN interface (either by port number or interface name); the remaining switch ports are set as LAN ports. The controller also implements a gateway. Both the subnet range and the gateway IP address are statically assigned through VIAS configuration, and VIAS divides the address space of nested VMs from the cloud providers' address space. When packets are sourced from the LAN address range and destined beyond the gateway (i.e. the destination address is out of the range of LAN subnet), the controller programs a flow rule in the OpenFlow switch to perform full-cone NAT mappings.

For example, as in Figure 3, assume a nested nVM with IP address 192.168.1.2 tries to access a public server 4.2.2.2 


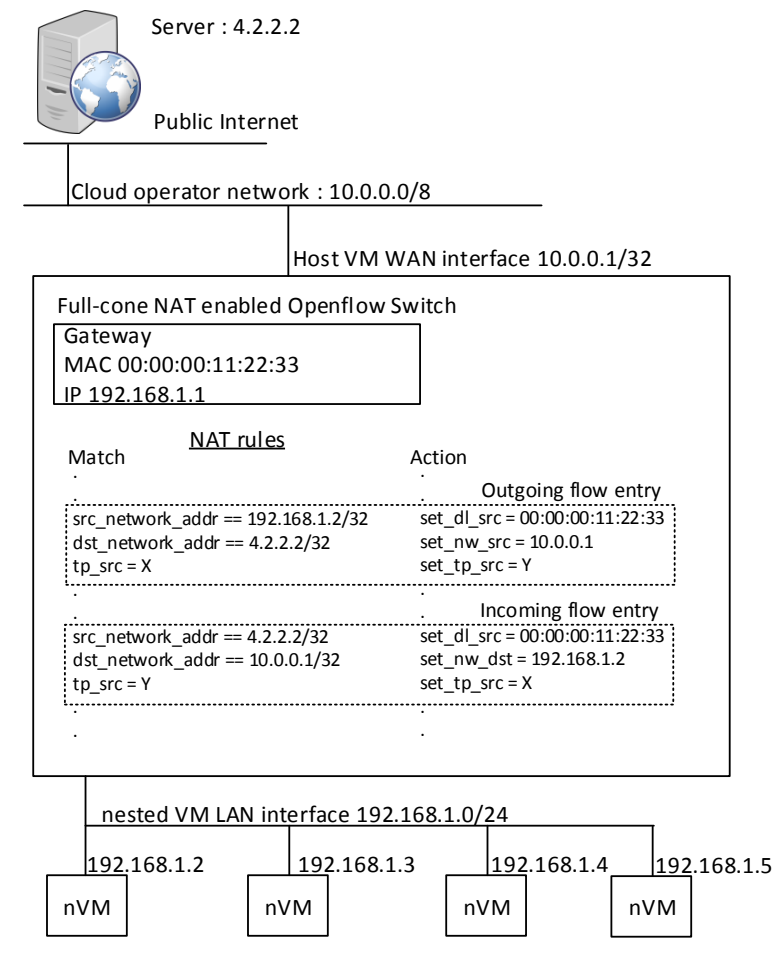

Figure 3: The VIAS SDN switch is programmed using OpenFlow to support NAT translation, in addition to "upcalls" to the overlay datapath and overlay bypass rules. This allows nVMs to access public Internet nodes through a layer-3 NAT gateway, in addition to exposing a virtual layer-2 network across all nodes connected to the overlay. In the example, an IP packet sent by nVM 192.168.1.2 to public server 4.2.2.2 triggers the programming of NAT rules by the VIAS SDN controller.

through TCP with source port number X. Initially, the destination address of the very first packet does not match any existing flow rules in the switch. Note that this controller supports MAC address learning, such that it can learn and map each nVM MAC address to its respective port. Then, the metadata of this first packet is forwarded to the SDN controller through the OpenFlow API of ofp_packet_in. The SDN controller then checks the destination address; it can determine that the destination 4.2.2.2 is not in its LAN segment, but instead across the gateway. The SDN controller then randomly chooses port number $\mathrm{Y}$ from an available port number pool (which is also configurable). Finally, it makes two flow entries: one is an outgoing flow entry for streams from nested nVM to the public Internet, and the other is an incoming flow entry for the reverse stream. This can be done with series of ofp_match and ofp_actions.

Continuing in the example, the outgoing flow entry translates address from 192.168.1.2:X to 10.0.0.1:Y, replacing the source address of nested $n V M$ to its WAN interface. The incoming flow entry translates destination address from 10.0.0.1:Y to 192.168 .1 .2 : $\mathrm{X}$, and injects the packets to the switch port of destination nested VM. The same technique can be applied to UDP streams.

\begin{tabular}{|c|c|c|}
\hline \multicolumn{2}{|c|}{ Bit 0} & \\
\hline \multirow{4}{*}{$\begin{array}{l}\text { Public } \\
\text { Network } \\
\text { Header }\end{array}$} & IP Header & 4 bytes \\
\hline & UDP Header & 2 bytes \\
\hline & Source IPOP UID & 20 bytes \\
\hline & Destination IPOP UID & 20 bytes \\
\hline \multirow{3}{*}{$\begin{array}{l}\text { Overlay } \\
\text { Network } \\
\text { Header }\end{array}$} & Ethernet Frame & 14 bytes \\
\hline & IP Header & \multirow{2}{*}{ Max 1280 bytes } \\
\hline & Payload & \\
\hline
\end{tabular}

Figure 4: Packet encapsulation in IPOP.

Since the absence of ICMP echo identifier field in match of OpenFlow specification, ICMP protocol, which is widely used for echo request/reply (ping) message, cannot be NATted. To this end, we forward every ICMP message to the controller, and NATting is performed in the SDN controller. While this approach increases the latency of ICMP messages, the functional behavior of the protocol is unaltered. Since ICMP is not used for traffic-intensive communications, this performance degradation is acceptable.

In the VIAS prototype, the SDN controller has been built upon the open-source RYU [26] SDN framework. The code (except the framework) is approximately 800 lines of Python code. For full backward compatibility, our implementation is based on OpenFlow specification version 1.0.

\subsection{Overlay datapath and Overlay Controller}

VIAS builds upon the IPOP codebase as a basis for the overlay datapath. In its current architecture, IPOP comprises of a packet processing/overlay datapath binary, and an overlay controller, which communicate through the TinCan API [12]. In essence, VIAS extends the IPOP overlay controller to support SDN bypass processing - we employ these two modules in VIAS as explained in Section 3.

In IPOP, nodes create direct P2P overlay links to tunnel virtual network traffic using the ICE protocol [24]. In order to bootstrap these direct P2P overlay links, an XMPP server is employed to assist in exchanging messages containing candidate endpoint information of peers - including the outer- and inner-most transport pairs, if nodes are behind multiple NATs. During this process, IPOP's overlay datapath module opens UDP ports ("hole-punching") on NATs to create P2P tunnels. Subsequently, peers communicate using these assigned UDP transports. The IPOP overlay header consists of two fields, which are the source and destination UIDs of overlay sender and receiver. The outer UDP and IP header are placed before the IPOP headers, resulting in the overall packet structure as shown in Figure 4.

In VIAS, each IPOP overlay link is considered as an OSI layer 2 tunnel. Each IPOP link is mapped such that multiple MAC addresses are bound to a link - which is akin to a layer 2 switch's MAC addresses bound to a port. The layer-2 overlay implements learning of MAC addresses by handling ARP request/reply messages in its LAN. IPOP checks the destination MAC address of the frame and injects the frame to the corresponding link. To bind the virtual network to VMs, IPOP uses virtual devices, including Linux bridge and Open vSwitch[17]. The tap interface is attached to this bind- 


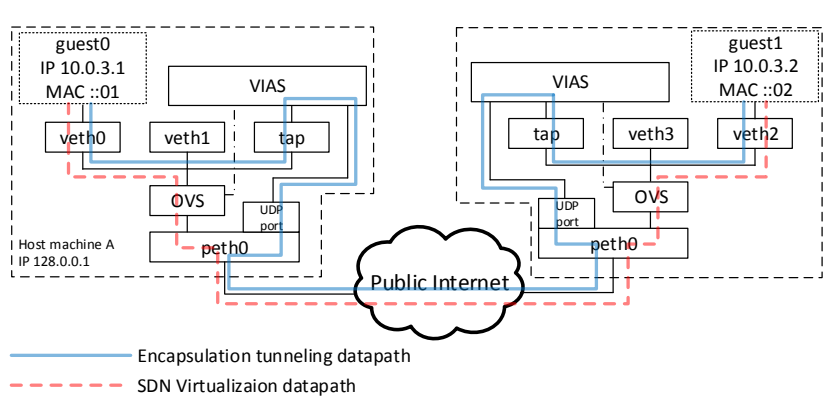

Figure 5: Packet datapath alternatives in VIAS. Initially, packets flow through the encapsulation tunneling datapath (solid line), through the overlay datapath module. Once a bypass flow is installed by the SDN controllers on both endpoints, the faster SDN virtualization datapath (dashed line) is used.

ing device and works as a bridge to the remote LAN virtual network.

For example, consider the usage scenario of Figure 5 . The illustration shows two hosts running VIAS-enhanced IPOP. Each host contains multiple nested VMs (containers) with virtual network interfaces (veth\#) attached to the Open vSwitch device. These guests are in the same layer 2 network, and are NATted by the host network stack to the physical interface (peth0).

When guest0 (veth0) attempts to send IP packets to guest1 (veth2), it first broadcasts an ARP request message. The VIAS-IPOP overlay datapath module picks this message through the tap device, and handles it as follows. First, the ARP message is encapsulated (Figure 4) and forwarded to all overlay links. At this stage, different overlay multicast approaches can be implemented by the overlay controller, depending on the overlay's topology. All the overlay nodes receiving the overlay-broadcast packet (e.g. the right-hand side of Figure 5) decapsulate the message and broadcast it to its L2 network (using the tap device). If there is no destination matching the ARP request message, the message is simply dropped. If the destination is in the network (e.g. veth2 in Figure 5), an ARP reply message is created by the guest, and the reply is sent back (with an unicast message) to the sender (e.g. guest0).

As part of this process, the overlay maps the MAC address it learned to the corresponding overlay link. All unicast MAC address frames captured by the overlay look up this mappings to determine along which overlay link to forward. VIAS mode can dynamically accommodate overlay topology changes by updating an MAC address and its overlay link bindings upon detecting an ARP frame. If we consider a usage scenario of VM migration from one host in a provider to another host in a different provider, the process can be automatically handled; there needs to be no network administrator involvement, since the ARP message from the migrated node itself incur updates the MAC-overlay link mapping on deployed overlay network.

\subsection{Software-defined Overlay Bypass}

As described in the previous section, the overlay virtualization process places several sources of overhead. At first, there is the transition overhead of context switch between network kernel to user space. It also requires multiple copy operations when it sends packet from network kernel to user space. Moreover, since there is the need for additional prepending of overlay headers, the MTU size is smaller than that of the physical network. This overhead is the price paid to create overlay virtual networks linking $n V M s$ across multiple providers, because tunneling, NAT traversal and encryption is required for virtual private networking. However, this overhead can be mitigated for nVMs within the same provider. To accomplish this, we bypass this encapsulation process on traffic-intensive TCP/UDP flows, as follows.

VIAS detects a traffic-intensive stream by monitoring traffic on overlay links, and can determine if an overlay path is possible by inspecting the overlay endpoints of a link whether they are within the same network. When VIAS determines that a bypass link should be created, it first allocates and assigns an available TCP (or UDP) port number pair for the outer address space. Then it establishes a mapping of inner address range to the outer address range with its port number. In OpenFlow, this can be programmed with a single flow add API call with ofp_flow_mod along a set of ofp_match and ofp_action.

On matched packet on ofp_match, the VIAS controller performs actions OFPAT_SET_DL_SRC and

OFPAT_SET_DL_DST to replace the MAC address of nested VM and Open vSwitch to physical Ethernet (peth) and gateway of outer address space. Then it performs actions OFPAT_SET_NW_SRC and OFPAT_SET_NW_DST to replace inner IP addresses to outer address space. Finally, actions OFPAT_SET_TP_SRC and OFPAT_SET_TP_DST replace the inner transport number to outer transport number. The incoming traffic takes the same steps, but changes from public address space to private address space.

To illustrate this behavior, consider the example of a TCP stream depicted in Figure 6 alongside the flow rule example of Table 1 . The example uses the private address space 10.0.3.0/24 for the inner address space for nested VMs, and public network addresses 128.0.0.0/24 (note that it is also possible to use private addresses for the outer address space, since VIAS supports NAT traversal). Initially, packets stream through encapsulation overlay datapath, VIAS prepending headers every packet. At certain threshold of traffic, VIAS triggers SDN bypass. First it extracts metadata of the stream, including the source/destination IP address (10.0.3.X) and port number (4000X). Then VIAS allocates and assigns an available port number (5000Y) to use as outer transport address. Next, VIAS programs inbound OpenFlow rules on the Open vSwitch SDN switch in host VM A. This inbounds rules translate outer transport (IP address and port number) to inner transport address. When VIAS programs an OpenFlow switch, it ensures the stream bypass rules have higher priority over other flow rules, so that the packet is not applied to other flow rules.

Soon after, VIAS sends a inter-controller RPC message through its $\mathrm{P} 2 \mathrm{P}$ overlay link to the peer overlay node, passing along JSON-formatted metadata of the stream. This metadata sent through the inter-controller RPC API contains information such as outer transport address, inner transport address and transport type.

Upon receiving the message, the VIAS controller at host B programs inbound and outbound rules to its Open vSwitch 


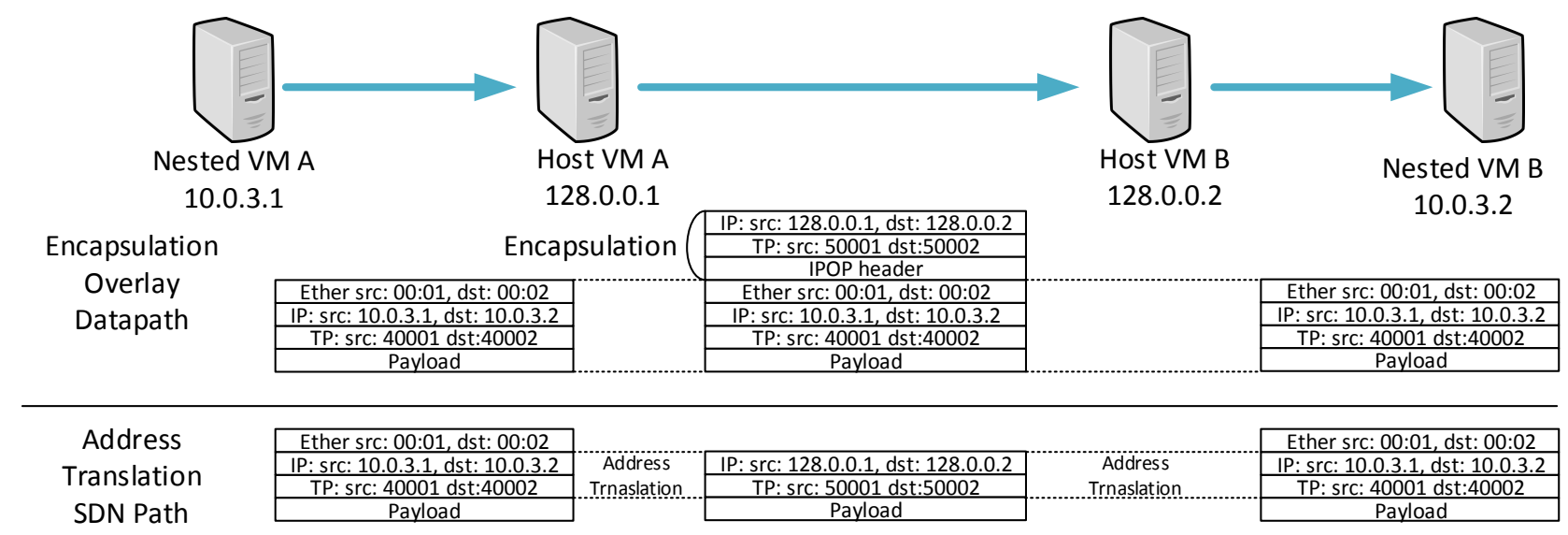

Figure 6: Virtualization comparison of datapath

Table 1: Stream Bypass Rule Example

\begin{tabular}{c|l|l|l}
\hline & & match & action \\
\hline \multirow{2}{*}{ local host } & outbound & $\begin{array}{l}\text { nw_src=10.0.3.1, nw_dst=10.0.3.2, } \\
\text { tp_src=40001, tp_dst=40002 }\end{array}$ & $\begin{array}{l}\text { set_nw_src=128.0.0.1, set_nw_dst=128.0.0.2, } \\
\text { set_tp_src=50001, set_tp_dst=50002 }\end{array}$ \\
\cline { 2 - 4 } & inbound & $\begin{array}{l}\text { nw_src=128.0.0.2, nw_dst=128.0.0.1, } \\
\text { tp_src=50002, tp_dst=50001 }\end{array}$ & $\begin{array}{l}\text { set_nw_src=10.0.3.2, set_nw_dst=10.0.3.1, } \\
\text { set_tp_src=40002, set_tp_dst=40001 }\end{array}$ \\
\hline remote host & outbound & $\begin{array}{l}\text { nw_src=10.0.3.2, nw_dst=10.0.3.1, } \\
\text { tp_src=40002, tp_dst=40001 }\end{array}$ & $\begin{array}{l}\text { set_nw_src=128.0.0.2, set_nw_dst=128.0.0.1, } \\
\text { set_tp_src=50002, set_tp_dst=50001 }\end{array}$ \\
\cline { 2 - 5 } & inbound & $\begin{array}{l}\text { nw_src=128.0.0.1, nw_dst=128.0.0.2, } \\
\text { tp_src=50001, tp_dst=50002 }\end{array}$ & $\begin{array}{l}\text { set_nw_Src=10.0.3.1, set_nw_dst=10.0.3.2, } \\
\text { set_tp_src=40001, set_tp_dst=40002 }\end{array}$ \\
\hline
\end{tabular}

SDN switch. The VIAS controller at host B then sends an acknowledgement RPC to host A through the overlay link, so that it makes sure that the outbound rule in local host is programmed only after all the other rules programmed. This ordering of events needs to be enforced to avoid packet loss during the setup of bypass rules. If we set local outbound rule before the other flow rules, the stream will have packets silently discarded by the SDN switch because of no matching rules. Finally, right after the final outbound rules are programmed in OpenFlow switches, the stream bypasses encapsulation and transfers packets through SDN switches on both endpoints.

This approach can be seen as an approach akin to NATs, as it provides the ability to map and translate addresses. However, unlike the conventional use of NAT, where each individual NAT is independently controlled, this scheme orchestrates the programming of mappings across controllers on both peer endpoints. VIAS essentially uses overlay link as control channels for coordination among the two peer SDN controllers to establish NAT mappings simultaneously across endpoints, allowing both nodes behind NATs to have a direct SDN flow that bypasses the overlay.

\section{EVALUATION}

In this section, we evaluate VIAS from three different perspectives. Firstly, in addition to providing overlay networking and bypass paths, VIAS acts as an OpenFlow-programmed
SDN bridge and NAT for nested VMs. Typically, nested VMs create virtual network interfaces bound to a host Linux bridge and NAT behavior is implemented using iptables. To evaluate the VIAS SDN bridge/NAT, we compare the performance of Open vSwitch-based VIAS to the native Linux bridge/NAT implementation. The second evaluation considers the throughput delivered by VIAS for TCP streams between nested VMs within and across cloud providers. Thirdly, we use an application-layer benchmark (Redis) to evaluate end-to-end VIAS performance. For all experiments, we use Ubuntu Linux 14.04 LTS hosts provisioned from clouds, software SDN switches (Open vSwitch version 2.0.2), and LXC containers [14] inside the host to create nested instances. VIAS is implemented as extensions to IPOP 15.01 using RYU as a framework for OpenFlow handling.

\subsection{VIAS Open vSwitch bridge/NAT}

As pointed out in Section 4.1, OpenFlow is not capable to program flow rules to handle NATting of ICMP echo request/reply. Consequently, all ICMP echo request/reply packets are forwarded by the SDN switch to the VIAS controller: the controller itself handles NAT for ICMP packets by making an entry in a local table for every outgoing ICMP echo request message, using the ICMP echo identifier field as a key to this table. Even though the VIAS controller runs in the same host as Open vSwitch, ICMP handling incurs overheads.

We compare Open vSwitch NAT overhead with the native 
Table 2: ICMP and TCP performance comparison between Linux NAT and NAT featured openvswitch of VIAS

\begin{tabular}{l|c|c|c|c|c}
\hline \multicolumn{2}{c|}{ Test case } & \multicolumn{2}{c|}{ host VM } & \multicolumn{2}{c}{ guest VM } \\
\cline { 3 - 6 } \multicolumn{2}{c|}{} & ICMP & TCP & ICMP & TCP \\
\hline \multirow{4}{*}{ S1 } & native & $0.487 \mathrm{~ms}[ \pm 0.104]$ & $5.24 \mathrm{Gbps}[ \pm 0.421]$ & $0.569 \mathrm{~ms}[ \pm 0.0611]$ & $4.09 \mathrm{Gbps}[ \pm 0.406]$ \\
\cline { 2 - 6 } & Open vSwitch & $7.76 \mathrm{~ms}[ \pm 0.790]$ & $4.87 \mathrm{Gbps}[ \pm 0.577]$ & $7.76 \mathrm{~ms}[ \pm 0.686]$ & $3.86 \mathrm{Gbps}[ \pm 0.518]$ \\
\cline { 2 - 6 } & percent change & $1493 \%$ & $-7.06 \%$ & $1264 \%$ & $-5.62 \%$ \\
\hline \multirow{3}{*}{ S2 } & native & $41.7 \mathrm{~ms}[ \pm 0.269]$ & $547 \mathrm{Mbps}[ \pm 10.4]$ & $41.7 \mathrm{~ms}[ \pm 0.514]$ & $411 \mathrm{Mbps}[ \pm 85.6]$ \\
\cline { 2 - 6 } & Open vSwitch & $49.2 \mathrm{~ms}[ \pm 1.35]$ & $527 \mathrm{Mbps}[ \pm 48.9]$ & $49.3 \mathrm{~ms}[ \pm 0.978]$ & $398 \mathrm{Mbps}[ \pm 83.1]$ \\
\cline { 2 - 6 } & percent change & $17.9 \%$ & $-3.80 \%$ & $18.2 \%$ & $-3.16 \%$ \\
\hline
\end{tabular}

Standard deviation is shown in square brackets

Linux bridge/NAT implemented by iptables in Table 2. In the experiment, host VMs are deployed in the CloudLab IGDDC Cluster in Utah and are provisioned with $862 \mathrm{MB}$ of memory. We run 50 ping tests (ICMP echo) and 10 iperf throughput (TCP) tests and report the arithmetric mean (and standard deviation), considering a client in CloudLab and two different servers. One server (S1) resides in the same CloudLab cluster. The other server (S2) is provisioned as an m1.small instance with $2 \mathrm{~GB}$ memory in the Chameleon cloud [3] in Texas.

As the results show, a latency overhead of about $7 \mathrm{~ms}$ is incurred in ICMP NAT handling, irrespective of the network distance to the server. This is because the overhead mostly comes from the inter-process communication between between Open vSwitch and the VIAS controller, and kernel/user context switch. This overhead is acceptable, as the ICMP echo message is typically used for network reachability checks and not for latency-sensitive applications.

The TCP iperf test results shows that the bridge/NAT throughput degrades about 3-7\% compared to native Linux iptables. As Open vSwitch developers argue that the TCP performance is equivalent to that of Linux bridge [19], the slight performance degradation observed is due to the NAT rules programmed by VIAS. The NAT rules in the native Linux case are set by iptables and executed in Linux network kernel, while the VIAS NAT is implemented by flow rules inside Open vSwitch.

\subsection{Microbenchmarks}

This experiment evaluates the performance of VIAS for virtual network communication among nested VM instances. To this end, we have deployed VIAS on multiple cloud service platforms, including commercial and academic clouds. This allows the evaluation of functionality and performance for intra- and inter-cloud deployments across various geographical locations. We demonstrate that the nested containers separated by multiple NATs across multiple clouds are successfully connected by a virtual layer- 2 network, and evaluate the performance of ARP, TCP, and ICMP protocols.

The following test cases are considered. The first case (CC) uses two Xen VMs deployed on CloudLab [4]. Each VM is provisioned with $862 \mathrm{MB}$ memory on CloudLab IGDDC. The second case (AA) uses two Amazon EC2 instances of type t2.medium in the same zone (Oregon). Although Amazon does not provide specifications of network throughput of $\mathrm{t} 2$.medium (only mentioning that its low to moderate), based on our link test in Figure 7, the performance levels are commensurate to an $1 \mathrm{Gbps}$ Ethernet link. The third case (GG) uses two Google compute engine instances of n1-standard-1 at US central zone. Host physical machine was provisioned on $\operatorname{Intel}(\mathrm{R}) \mathrm{Xeon}(\mathrm{R}) \mathrm{CPU} @$ $2.30 \mathrm{GHz}$ and $3.7 \mathrm{~GB}$ memory. In the fourth case (AA_dz), we run experiments also with t2.medium Amazon EC2 instances, but with VMs distributed across two availability zones (N. Virginia and Oregon) for comparison. Finally, the fifth case (AG_dz) considers two instances deployed across two different cloud service providers. One instance is on Amazon EC2 (t2.medium) at Oregon and the other instance is on Google compute engine (n1-standard-1) at US central.

Column physical is the latency between host VMs. The overlay column shows the latency between nested VMs. The traffic streams through overlay datapath from Linux bridge to tap device to P2P tunnel. The VIAS column shows the latency of overlay datapath with Open vSwitch and tap device. Note that, for the VIAS column, ARP and ICMP is forwarded to SDN controller, incurring additional latency.

ARP: The ARP latency is measured using iputils-arping and is shown on Table 3 . The test is repeated 50 times, and the arithmetic mean and standard deviation are reported. The results for the AA, CC and GG case show that the overhead of ARP handling in overlay is less than $1.5 \mathrm{~ms}$, while in VIAS, the latency overheads are in the range of 4-24ms, due to inter-process communication and SDN controller processing. Surprisingly, the results show an exceptionally long latency of ARP across host Xen VMs in one particular environment $\mathrm{CC}$ - the overlay and VIAS latencies are smaller than physical network. While we were not able to definitively determine the reason for this behavior, one observation is that the VIAS and the overlay paths use UDP as the protocol, whereas arping on the host VMs uses the ARP protocol. It is possible that the CloudLab platform handles ARP and UDP in different ways - since ARP traffic is relatively infrequent, the effects of delay in overall network performance are typically not significant. The ARP measurements in the AA case show that physical latency is lowest.

Naturally, geographically separated nested VMs (AA_dz and AG_dz) exhibit longer ARP latencies due to network distance; this is observed in the results summarized in the overlay column. Note that because the AA_dz and AG_dz instances are not in the same LAN segment, ARP traffic in the physical network is not supported, hence the physical column shows N/A. Furthermore, we could not evalu- 
Table 3: ARP and ICMP latency comparison among conventional Linux implementation, overlay datapath and VIAS

\begin{tabular}{c|c|c|c|c|c|c}
\hline \multirow{2}{*}{$\begin{array}{c}\text { Test } \\
\text { case }\end{array}$} & Physical & Overlay & VIAS & Physical & Overlay & VIAS \\
\cline { 2 - 7 } CC & $432[ \pm 243]$ & $1.47[ \pm 0.130]$ & $24.4[ \pm 0.102]$ & $0.954[ \pm 0.121]$ & $1.17[ \pm 0.0825]$ & $15.4[ \pm 1.20]$ \\
AA & $0.693[ \pm 0.0357]$ & $1.42[ \pm 0.0805]$ & $4.00[ \pm 0.120]$ & $0.559[ \pm 0.132]$ & $0.970[ \pm 0.149]$ & $5.33[ \pm 0.197]$ \\
GG & N/A & $0.4207[ \pm 0.126]$ & $4.63[ \pm 0.372]$ & $0.421[ \pm 0.126]$ & $0.697[ \pm 0.0843]$ & $6.22[ \pm 0.409]$ \\
AA_dz & N/A & $84.5[ \pm 0.243]$ & N/A & $84.6[ \pm 0.400]$ & $92.5[ \pm 0.169]$ & N/A \\
AG_dz & N/A & $49.7[ \pm 0.116]$ & N/A & $50.7[ \pm 0.101]$ & $50.2[ \pm 0.379]$ & N/A \\
\hline
\end{tabular}

CC: cloudlab AA:Amazon GG:Google compute engine AG:Amazon-Google dz:different zone. Standard deviation is shown in square brackets

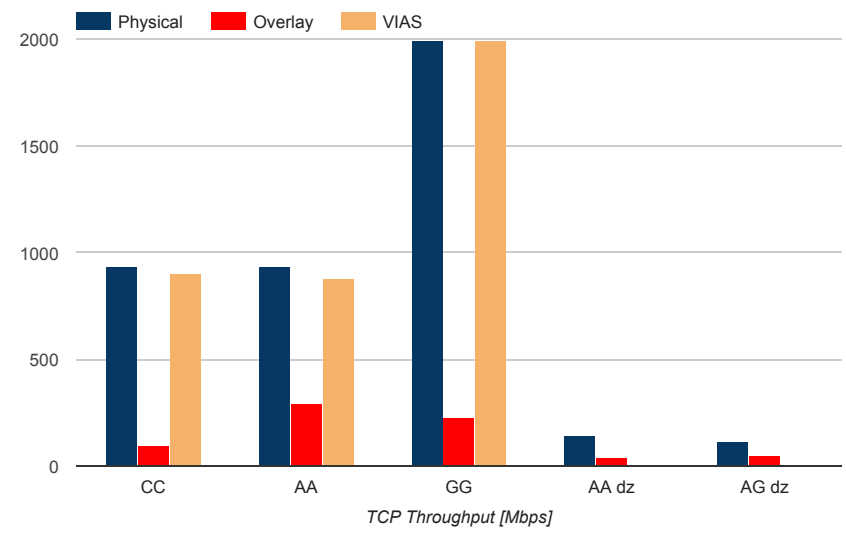

Figure 7: TCP performance comparison among physical, overlay datapath and VIAS bypass virtualization scenario

ate ARP in GG case, because Google compute engine does not provide layer 2 abstraction among instances deployed in the same zone, and thus the ARP protocol does not work. Nonetheless, the results demonstrate that, regardless of providers blocking L2 traffic within or across clouds, VIAS can present a L2 virtual network to nested VMs.

ICMP: ICMP echo latency is measured using the Linux ping command. We repeated the test 50 times, and report the arithmetic mean and standard deviation of the latency. The trend is similar with the ARP latency. The latency overhead is of the order of a few milliseconds across all test cases. Since the overhead only concerns in local kernel/user boundary crossing and local socket interface, the nested VM latency is not a function of the physical network's latency, but rather a constant overhead at the endpoints.

TCP: In Figure 7, TCP throughputs with different configurations between nested VMs across different cloud services are summarized. The iperf tool is used to test the maximum TCP throughput; tests were repeated 10 times, and the arithmetic means are shwon. For the physical column, iperf was executed on the host VM, while for the overlay and SDN bypass columns, iperf was executed on the nested VMs. Note that the VIAS bypass is not shown for experiments across cloud data centers, where tunneling is used instead.

The results show that encapsulation in user space has its peak throughput at around a few of hundreds Mbps, regardless of link layer bandwidth. This overlay performance is a function of the host processor's performance, and overheads associated with packet handling and copying and $\mathrm{O} / \mathrm{S}$ kernel-user context switch. In contrast, SDN virtualization achieves over $94 \%$ of the throughput of the physical network when endpoints are in the same data center (cases CC, AA and GG). Note that this experiment considers a software Open vSwitch that runs on the host VM, and does not use any assist from SDN hardware - though in principle VIAS can also program hardware-layer SDN switches, if available. Nonetheless, by eliminating $\mathrm{O} / \mathrm{S}$ context switch and overlay packet handling through the VIAS SDN overlay bypass results in substantial performance improvements for network virtualization.

As described in Section 4.3, VIAS detects traffic-intensive TCP streams at runtime, and then automatically inserts bypass rules in the SDN fabric. Prior to completion of flow rule, TCP streams traverse the overlay datapath. Thus, there is a latency involved in the coordinated programming of flow rules in the SDN switch of both peer endpoints. This latency is a function of the round trip time between host VMs: we have measured the SDN bypass setup latency to be $13.5 \mathrm{~ms}, 12.3 \mathrm{~ms}$ and $2.63 \mathrm{~ms}$, respectively, in the CC, AA and GG cases. This latency is measured using the difference between the first packet and the last packet in the encapsulation path of each TCP stream.

\subsection{Application Test}

In this section, we show the virtualization performance at the application layer, using a round-trip latency sensitive application: Redis [22], a NoSQL, memory-based and keyvalue data structure storage system that is widely used. In the experiment, two host VMs are deployed in the CloudLab IG-DDC Cluster, provisioned with $862 \mathrm{MB}$ of memory. The test is done with the version 3.0.6 of Redis. The key length is 10 Bytes long and value length are set to 50 Bytes long, a common usage pattern of Redis [6]. For each run, clients make 1 million queries (50\% sets and 50\% gets). Every set packet size is 93 Bytes, and get return packets from the get are 36 and 57 Bytes, respectively.

Figure 8 shows the results of physical, overlay datapath, and VIAS SDN bypass cases. One important observation needs to be made - that the "Physical" case is one where when both Redis server and clients runs on the host VM, while in overlay datapath and SDN bypass cases, those run on nested containers. Each thread setup a single TCP stream with Redis server.

The throughput of VIAS SDN bypass is on par with the 


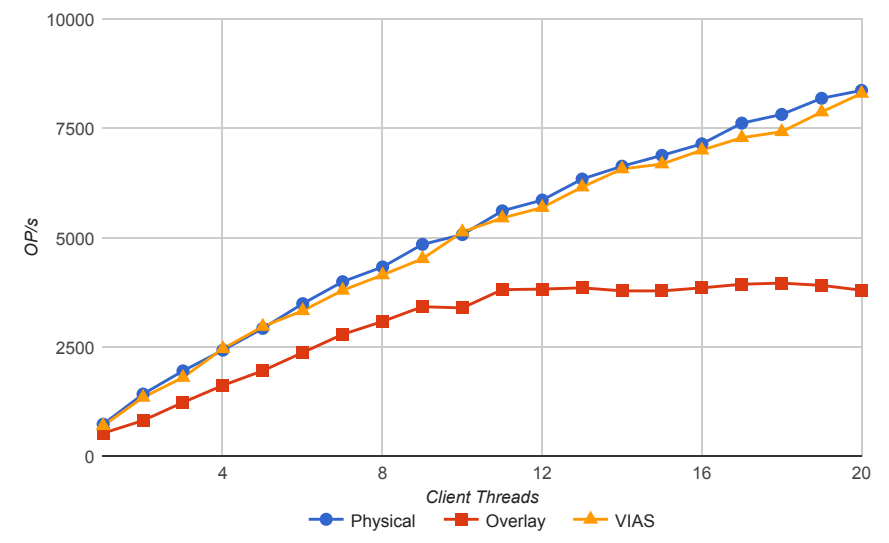

Figure 8: Redis simulation.

physical case, proportionally increasing the throughput as physical case with the increasing number of thread counts. On the contrary, overlay datapath throughput saturates around $4 \mathrm{KOP} / \mathrm{S}$. The experiment shows that VIAS bypass performs significantly better than overlay encapsulation, and that it is capable to bypass multiple TCP streams simultaneously.

\section{RELATED WORK}

The idea of building user-level virtual networks for Grid/cloud computing dates back to systems including Violin [11], VNET [29], and IPOP [8]. Violin proposed overlay virtual networks providing the abstraction of separate layer-2 networks for tenants. Violin was implemented without requiring any modifications on the VMM or the hosting network infrastructure, not only providing flexible user configurable network environment, but also reducing the threat of security risk from the host. VNET addressed a similar problem, focusing on the ability to inter-connect virtual machines across providers. This was accomplished by running VNET "proxies" on endpoints (e.g. VMs) at different sites, and tunneling L2 traffic over TCP/TLS links.

A limitation of these systems is performance - nowadays, cloud network infrastructure performance is $1 \mathrm{Gbps}$ or higher, making it challenging to deliver high-performance network virtualization at the application layer on commodity CPUs. VNET/P [34] addresses the performance gap by implementing a kernel module for layer 2 virtualization of guest VMs inside the VMM increasing the performance of virtual networks substantially by moving virtualization process from user space to a VMM module. It showed that it can achieve line speed performance virtualization in 1 Gbps network and $78 \%$ in 10 Gbps network. However, it requires changes to the VMM, which hinders deployment of this technique. VIAS seeks to also bypass user-level processing, but does so while reusing existing, unmodified systems by leveraging software SDN switches. Our experiments have shown that it is possible to deploy VIAS in existing cloud infrastructures (Amazon EC2, Google compute engine, CloudLab) without requiring any changes to VMMs nor VM images - VIAS only requires user-level software to be installed.

Another approach to minimize context switch between kernel and userspace is Netmap [23]. It eliminates the copy operation by using shared buffer and metadata between kernel and user space, showing that it achieves 20x speedups compared to conventional APIs. However, netmap relies on its own custom kernel module, again hindering the ability to deploy on commodity systems.

Instead of providing full virtual network to the users, VirtualWire [33] takes advantage of connect/disconnect primitives, which are assumed to be exposed by the cloud service provider. Their implementation requires changes to a Xen-blanket hypervisor, while VIAS can be deployed without changes to the VMM. Furthermore, VIAS makes no assumption about the primitives exposed by a provider to manage connectors - VIAS overlay links can be established even when cloud instances are constrained by NATs.

NVP [13] is closely related as it also shows combined version of tunneling and SDN fabric. It provides REST APIs to the tenants to expose network virtualization capabilities. Network elements such as switches and ports are presented to the tenants, and tenants build topology of their network. Then, tunnels and flow rules are created and programmed by NVP to each hardware and software OpenFlow switches to forward packets among VMs deployed intra and inter clouds. However, NVP is designed to support multiple tenants in a single cloud provider. Unlike VIAS, its techniques do not support inter-cloud network virtualization.

\section{CONCLUSIONS AND FUTURE WORK}

This paper presented the novel architecture of VIAS, and demonstrated its ability to automatically provide fast virtual network paths within a cloud provider via coordinated programming of SDN switches, while retaining the ability to dynamically establish inter-cloud virtual private network tunnel. The main contribution of this paper is a novel userlevel approach to distributed, coordinated control of overlay and SDN controllers, supporting private inter-cloud and high-performance intra-cloud network virtualization flows.

VIAS leverages existing system-level VMM/kernel software without modifications, and has been demonstrated to work by extending existing overlay software (IPOP) and SDN platforms (RYU, Open vSwitch) in realistic cloud computing environments, including Amazon EC2 and Google compute engine. Results showed that VIAS can provide flexible layer 2 virtual network, in particular to nested virtualization environments, where tenants deploy containers across multiple providers.

While this paper has only quantitatively evaluated the use of VIAS with software virtual switches, the use of the OpenFlow standard allows VIAS to tap into hardware SDN resources, if available. In future work, we plan to offload the processing overhead of network virtualization from software to hardware OpenFlow devices.

\section{ACKNOWLEDGMENTS}

This material is based upon work supported in part by the National Science Foundation under Grants No. 1527415, 1339737 and 1234983, and by the Department of Defense award W911NF-13-1-0157. Any opinions, findings, and conclusions or recommendations expressed in this material are those of the author(s) and do not necessarily reflect the views of the National Science Foundation or the Department of Defense. The authors acknowledge the use of NSFCloud resources (CloudLab and Chameleon) for experiments. 


\section{REFERENCES}

[1] AWS SDK for Python (Boto3). https://aws.amazon.com/sdk-for-python/.

[2] M. Ben-Yehuda et al. The turtles project: Design and implementation of nested virtualization. In Proceedings of the 9th USENIX Conference on Operating Systems Design and Implementation, OSDI'10, pages 1-6, Berkeley, CA, USA, 2010. USENIX Association.

[3] Chameleon. https://www.chameleoncloud.org/.

[4] CloudLab. http://cloudlab.us/.

[5] E. Deelman et al. Pegasus, a workflow management system for science automation. In Future Generation Computer Systems Volume 46, pages 17-35, 2015.

[6] W. Felter et al. An updated performance comparison of virtual machines and linux containers. IBM technical report RC25482 (AUS1407-001), 2014.

[7] A. Fishman et al. Hvx: Virtualizing the cloud. In Presented as part of the 5th USENIX Workshop on Hot Topics in Cloud Computing, Berkeley, CA, 2013. USENIX.

[8] A. Ganguly, A. Agrawal, P. O. Boykin, and R. Figueiredo. Ip over $\mathrm{p} 2 \mathrm{p}$ : enabling self-configuring virtual ip networks for grid computing. In Proceedings 20th IEEE International Parallel Distributed Processing Symposium, pages 10 pp.-, April 2006.

[9] Google Cloud Platform. gcloud Tool Guide. https://cloud.google.com/sdk/gcloud/.

[10] E. Hernandez-Valencia et al. How will NFV/SDN transform service provider opex? Network, IEEE, 29(3):60-67, May 2015.

[11] X. Jiang and D. Xu. Violin: Virtual internetworking on overlay infrastructure. In Parallel and Distributed Processing and Applications, volume 3358 of Lecture Notes in Computer Science, pages 937-946. Springer Berlin Heidelberg, 2005.

[12] P. S. Juste et al. Tincan: User-defined p2p virtual network overlays for ad-hoc collaboration. EAI Endorsed Transactions on Collaborative Computing, 14(2), 102014.

[13] T. Koponen et al. Network virtualization in multi-tenant datacenters. In 11th USENIX Symposium on Networked Systems Design and Implementation (NSDI 14), pages 203-216, Seattle, WA, Apr. 2014. USENIX Association.

[14] Linux Containers. https://linuxcontainers.org/.

[15] R. Mahy et al. Traversal Using Relays around NAT (TURN): Relay Extensions to Session Traversal Utilities for NAT (STUN). RFC 5389, April 2010.

[16] N. McKeown et al. Openflow: Enabling innovation in campus networks. SIGCOMM Comput. Commun. Rev., 38(2):69-74, Mar. 2008.

[17] Open vSwitch. http://www.openvswitch.org/.

[18] A. M. Oprescu and T. Kielmann. Bag-of-tasks scheduling under budget constraints. In Cloud Computing Technology and Science (CloudCom), 2010 IEEE Second International Conference on, pages 351-359, Nov 2010.

[19] B. Pfaff et al. The design and implementation of open vswitch. In 12th USENIX Symp. on Networked Systems Design and Implementation (NSDI 15), pages 117-130, Oakland, CA, May 2015. USENIX Assoc.
[20] G. J. Popek and R. P. Goldberg. Formal requirements for virtualizable third generation architectures. Commun. ACM, 17(7):412-421, July 1974.

[21] K. Razavi et al. Kangaroo: A Tenant-Centric Software-Defined Cloud Infrastructure. In Proc. of the IEEE International Conference on Cloud Engineering, Tempe, AZ, USA, United States, Mar. 2015.

[22] Redis. http://redis.io/.

[23] L. Rizzo. netmap: A Novel Framework for Fast Packet I/O. In 21st USENIX Security Symposium (USENIX Security 12), pages 101-112, Bellevue, WA, Aug. 2012. USENIX Association.

[24] J. Rosenberg. Interactive Connectivity Establishment (ICE): A Protocol for Network Address Translator (NAT) Traversal for Offer/Answer ProtocolsA Border Gateway Protocol 4 (BGP-4). RFC 5245, April 2010.

[25] J. Rosenberg et al. Session Traversal Utilities for NAT (STUN). RFC 5389, October 2008.

[26] Ryu SDN Framework. http://osrg.github.io/ryu/.

[27] T. Saad et al. Tunneling techniques for end-to-end VPNs: generic deployment in an optical testbed environment. In Broadband Networks, 2005.

BroadNets 2005. 2nd International Conference on, pages 859-865 Vol. 2, Oct 2005.

[28] I. Stoica et al. Chord: A scalable peer-to-peer lookup service for internet applications. In Proceedings of the 2001 Conference on Applications, Technologies, Architectures, and Protocols for Computer Communications, SIGCOMM '01, pages 149-160, New York, NY, USA, 2001. ACM.

[29] A. I. Sundararaj and P. A. Dinda. Towards virtual networks for virtual machine grid computing. In Proceedings of the 3rd Conference on Virtual Machine Research And Technology Symposium - Volume 3, VM'04, pages 14-14, Berkeley, CA, USA, 2004. USENIX Association.

[30] R. Uhlig et al. Intel virtualization technology. Computer, 38(5):48-56, May 2005.

[31] G. Wang and T. Ng. The impact of virtualization on network performance of amazon ec 2 data center. In INFOCOM, 2010 Proceedings IEEE, pages 1-9, March 2010.

[32] D. Williams et al. The xen-blanket: Virtualize once, run everywhere. In Proceedings of the 7th ACM European Conference on Computer Systems, EuroSys '12, pages 113-126, New York, NY, USA, 2012. ACM.

[33] D. Williams et al. VirtualWires for Live Migrating Virtual Networks across Clouds. IBM Research Report RC25378, 2013.

[34] L. Xia et al. VNET/P: Bridging the Cloud and High Performance Computing Through Fast Overlay Networking. In Proc. of the 21st International Symposium on High-Performance Parallel and Distributed Computing, HPDC '12, pages 259-270, New York, NY, USA, 2012. ACM.

[35] F. Zhang et al. Cloudvisor: Retrofitting protection of virtual machines in multi-tenant cloud with nested virtualization. In Proc. of the Twenty-Third ACM Symposium on Operating Systems Principles, SOSP '11, pages 203-216, New York, NY, USA, 2011. ACM. 